\title{
Les protéines de régulation de la mélanogenèse Génétique moléculaire des albinismes cutanés
}

La pigmentation - ou mélanogenèse - résulte des interactions de plusieurs gènes et de leurs produits, et conduit à la production des mélanines. Ces pigments spécifiques sont synthétisés par les mélanocytes, à l'intérieur d'organites spécialisés, les mélanosomes. La régulation de la mélanogenèse s'effectue à différents niveaux ; cependant, l'enzyme tyrosinase est principalement responsable du contrôle de la biosynthèse des mélanines. Chez la souris, des travaux antérieurs ont démontré la liaison du gène de la tyrosinase avec le locus albino. A ce jour, la structure de la protéine a été élucidée, son gène cloné, et différentes mutations impliquées dans les différentes formes d'albinisme ont été caractérisées chez l'homme et chez la souris. D'autres gènes codant pour des protéines apparentées à la tyrosinase et également impliqués dans la pigmentation ont été récemment isolés. La connaissance de leurs mécanismes d'action et de leurs interactions sera essentielle pour une meilleure compréhension des mécanismes de la mélanogenèse.

\section{Brigitte Bouchard}

\section{ADRESSE}

B. Bouchard : chargée de recherche à l'Inserm. Inscrm U. 312, hôpital Henri-Mondor, 51, avenuc du Maréchal-de-Lattrc-dc-Tassigny, 94010 Crétcil, France.

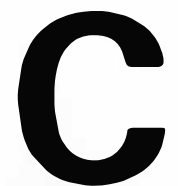

hez les mammifères, la pigmentation est un processus complexe qui résulte de la synthèse de pigments spécifiques, les

mélanines

Cette synthèse et l'ensemble des mécanismes qui la contrôlent constituent la mélanogenèse, dont l'étude fascine depuis fort longtemps les généticiens. En effet, chez l'animal, les travaux cherchant à ćlucider les lois qui déterminent la couleur du pelage ont débuté durant les premières années de ce siècle [1]. Alors que les bases anatomiques et biochimiques de la pigmentation ćtaient encore inconnues, ces premiers travaux établirent clairement que les différents pelages observés chez l'animal impliquent l'interaction de plusicurs gènes et de leurs produits, et sont, pour leur plus grande part, indifférents aux facteurs environnementaux.

Par la suite, les premières données démontrant les bases cellulaires de la pigmentation ont été obtenues. La mélanogenèse se produit dans les mélanocytes situés à la jonction dermo-épidermique qui repose sur la membrane basale, ou dans les follicules pileux, au niveau de la papille dermique [2]. Les mélanocytes sont des cellules hautement spécialisćes dont la caractéristique principale est la capacité de produire les mélanines. Ces cellules dérivent de la crête neurale, ćmergeant de la surface du tube 


\section{RÉFÉRENCES}

1. Castle WE, Gates WH, Recd SC, Law LW. Studics of a sizc cross in mice, II. Genetics $1910 ; 21: 310-23$

2. Rawles ME. Origin of the mammalian cell and its rolc in the pigmentation of hair In : Gordon M, cd. Pigment Cell Growth. New York: Academic Press, 1953 : 1-12.

3. Silvers WK. The Coat Colors of Mice. New York: Springer Verlag, 1979.

4. Hearing VJ, Jimencz M. Mammalian tyrosinase: the critical regulatory control point in melanocytc pigmentation. Int J Biochem $1987 ; 19: 1141-7$

5. Pomcrantz SHI. I--Tyrosinc 3,5'-3H assay for tyrosinase development in skin of new born hamsters. Science $1969 ; 164$. 838-43.

6. Kwon BS, Haq AK, Pomerantz SHL, Halaban $R$. Isolation and sequence of a cDNA clone for human tyrosinase that maps as the c-albino locus. Proc Natl Acad Sci USA $1987 ; 84: 7473-7$

7. Shibahara S, Tomita Y, Sakamura T, Nager C, Chaudhuri B, Muller R. Cloning and expression of cDNA cncoding mousc tyrosinasc. Nucleic Acids Res $1986 ; 14$ 2413-27

8. Yamamoto 11, 'I'akcuchi S, Kudo ' 1 ', Makino KK, Nakata A, Shinoda T, Takcuchi T. Cloning and scquencing of mousc tyrosinasc cDNA. Jpn J Genet 1987; 62 : 271-4.

9. Muller G, Ruppert S, Schmid E Schutz G. Functional cvidence of alternatively spliced tyrosinase genc transcripts. EMBO J $1988 ; 7$ : 2723-30.

10. Bouchard B, Fuller BF, Vijayasaradhi S, Houghton AN. Induction of pigmentation in mousc fibroblasts by transfection of human tyrosinasc cDNA. $J$ Exp Med $1989 ; 169: 2029-42$.

11. Bccrman F, Ruppert S, Hummler E, Bosch FX, Muller G, Ruther U, Schutz G. Rescuc of the albino phenotype by introduction of a functional tyrosinase genc into micc. $E M B O J 1990 ; 9$ : 2819-26.

12. Ruppert S, Muller G, Kwon B, Schutz G. Multiple transcripts of the mousc tyrosinase gene are generated by alternative splicing. EMBO J $1988 ; 7: 2715-22$.

13. Gicbcl L, Strunk KM, Spritz RA Organization and nucleotide sequences of the human tyrosinase gene and a truncated tyrosinasc-rclated scgment. Genomics 1991 ; $9: 435-45$

14. Barton DE, Kwon BS, Francke U. Human tyrosinase genc, mapped to chromosome 11 (q14-21) defincs sccond region of homology with mouse chromosome 7. Genomics $1988 ; 3: 17-24$. neural durant la fermeture des plis neuronaux. Les mélanines sont synthétisées à l'intérieur d'organites particuliers, les mélanosomes, constitués d'une matrice fibreuse contenue elle-même à l'intérieur d'une structure membranaire. La présence de ces organites n'est connue que dans les cellules appartenant à la lignée mélanocytaire et de leurs dérivés.

L'analyse génétique de la pigmentation, en particulier chez la souris, a permis d'établir l'existence de nombreuses mutations (plus de 140), qui affectent les différents loci $(>60)$ impliqués plus ou moins directement dans la détermination génétique de la couleur du pelage [3]. Certaines de ces mutations altèrent les fonctions de la pigmentation ou des fonctions liées à celles-ci, alors que d'autres ont des effets marqués sur le développement de l'animal.

La pigmentation est donc contrôlée à différents niveaux, chacun par un mécanisme bien précis. La migration des mélanocytes au cours du développement, la biogenèse des mélanosomes, la migration de ces derniers dans les kératinocytes environnants, représentent trois des niveaux où s'exerce un contrôle génétique que nous commençons maintenant à mieux comprendre.

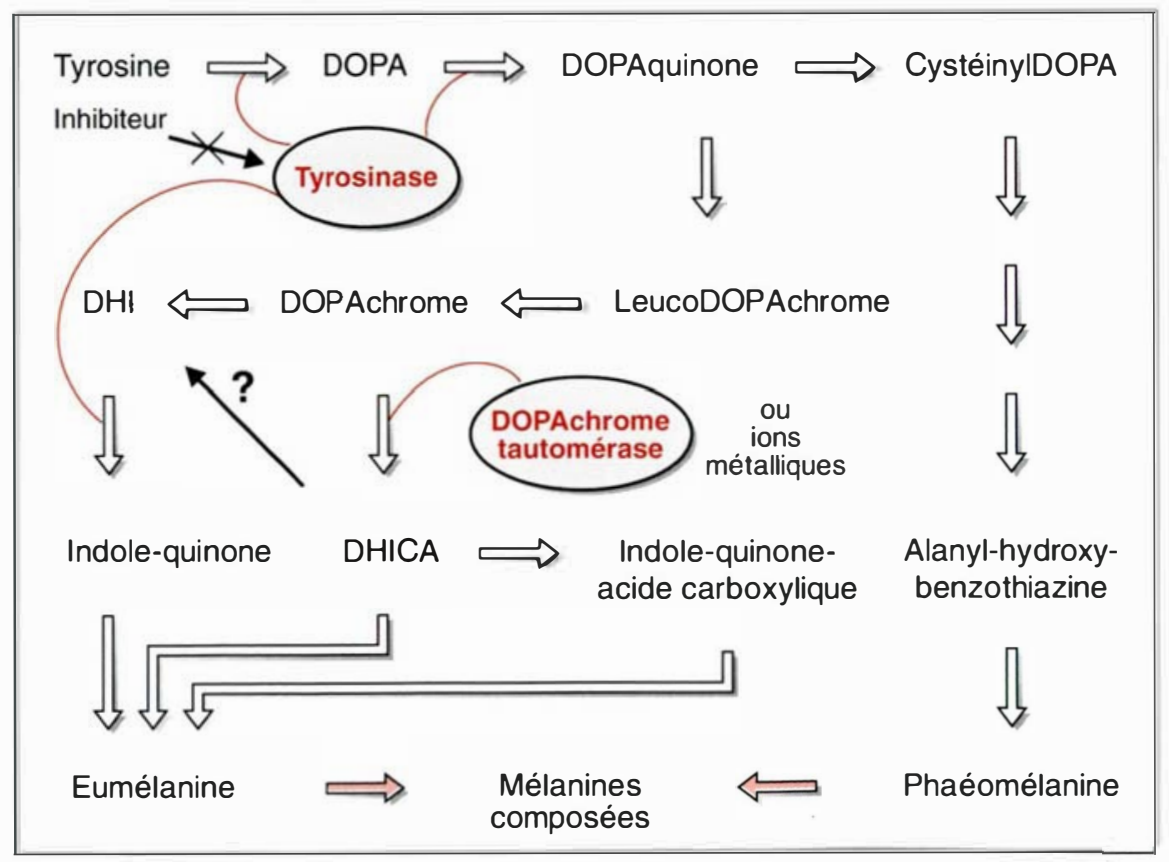

Figure 1. Synthèse des mélanines. DHI: dihydroxyindole : DHICA : dihydroxyindole carboxylic acid.

\section{Synthèse des mélanines}

Depuis fort longtemps, les biologistes savent qu'une enzyme, la tyrosinase (E.C.1.14.18.1) est le catalyseur responsable de la synthèse des mélanines chez les mammifères. Deux types de mélanine peuvent être produites, la phaeomélanine (de type jaune ou De nombreux facteurs interviennent dans le type et la quantité de mélanine synthétisée. Ainsi, chez la souris, la MSH (melanocyte stimulating hormone) peut augmenter le degré de pigmentation de façon remarquable. En revanche, chez l'homme, le rôle médiateur de la voie de l'AMPc semble plus important (figure 1). Cependant, il est clair que la tyrosinase joue un rôle essentiel dans le processus de synthèse des mélanines. Il s'agit d'un système monoenzymatique possédant plusieurs points de contrôle. En effet, de manière inhabituelle, la tyrosinase catalyse trois réactions dans la voie de synthèse des mélanines [4]. Tout d'abord, l'hydroxylation du substrat tyrosine en DOPA (3,4-dihydroxyphénylalanine), puis l'oxydation de la DOPA en DOPAquinone, et enfin l'oxydation de la dihydroxyindole en indole quinone. rouge) et l'eumélanine (de type noir). 
L'introduction de tests in vitro permettant de mesurer quantitativement la première de ces réactions, qui est aussi la plus significative, a permis d'analyser la tyrosinase sur le plan biochimique et de caractériser les étapes intracellulaires de sa biosynthèse [5].

\section{Données moléculaires sur la tyrosinase}

Les études biochimiques et moléculaires ont permis de déterminer la structure de l'enzyme tyrosinase. La molécule est constituée de plusieurs domaines distincts, dont trois régions riches en cystéines, et une région transmembranaire hydrophobe par laquelle la protéine s'intègre dans la membrane des mélanosomes (figure 2). Lors de son passage dans l'appareil de Golgi, la tyrosinase est glycosylée avant d'être déversée dans les mélanosomes, qui constituent son lieu d'action, par le biais de vésicules cytoplasmiques.

La tyrosinase étant l'enzyme clé de la mélanogenèse, l'on comprend aisément que des mutations affectant son gène pourraient conduire en particulier aux différentes formes d'albinisme. De nombreux efforts se sont donc portés vers l'isolement du gène de la tyrosinase au cours de ces dernières années. Différents laboratoires ont isolé et identifié des clones d'ADNc spécifiques de mélanocytes humains ou murins [6-9]. Ces différents clones présentent des structures très homologues et codent pour des protćines de poids moléculaires voisins $(70$ à $75 \mathrm{kDa})$, ont une spécificité d'expression dans les cellules mélanocytaires, et ont pour cela été proposés comme candidats correspondant au gène de la tyrosinase. Par ailleurs, des études génétiques antérieures, menées chez la souris, ont décrit plusieurs mutations du locus albino, dont les conséquences sont l'absence de mélanine, qui se manifeste par le pelage uniformément blanc caractéristique de ces animaux. Au niveau de l'ultrastructure cellulaire, on sait que les mélanocytes des animaux albino produisent des "prémélanosomes " incapables de se mélaniser. Néanmoins, ces observations avaient été insuffisantes pour déterminer si le locus albino correspondait au gène de la tyrosinase ou à un gène impliqué dans sa régulation.

L'isolement d'un clone d'ADNc situé sur le locus albino [6] a permis de démontrer son rôle exact dans la mélanogenèse en obtenant une preuve fonctionnelle. En effet, in vitro, la protéine codée par le locus albino possède à elle seule une activité tyrosinase. En premier lieu, il a été montré que la transfection de l'ADNc humain dans des cellules sans activité tyrosinase endogène (fibroblastes ou cellules Hela) permet à ces cellules de synthétiser l'enzyme sous forme active, capable d'induire la production de mélanine dans des cellules pourtant dépourvues de mélanosomes [10]. De façon complémentaire, les expériences de différents groupes ont montré que des souris transgéniques de souche albino exprimant l'ADNc de la tyrosinase présentent une augmentation radicale du degré de pigmentation [11].

Par la suite, la structure du gène de la tyrosinase a été déterminée. Ce gène est composé de cinq exons et de quatre introns (figure 3) [12]. Sa taille est de $70 \mathrm{~kb}$ chez la souris et est vraisemblablement identique chez l'homme. La taille de l'AR Nm correspondant est de 2,4 kb. Un premier niveau de régulation du gène de la tyrosinase se situe lors de l'épissage permettant l'excision des introns non codants. Environ 10 à $40 \%$ des ARN sont en effet des transcrits alternatifs, ce qui conduit à la traduction de protéines altérées. Le rôle éventuel de ces transcrits modifiés n'est pas connu ; ils pourraient éventuellement jouer le rôle d'inhibiteurs compétitifs, réglant ainsi l'expression de la tyrosinase. Il n'existe qu'une copie du gène de la tyrosinase dans le génome, mais l'existence d'un pseudogène constitué seulement des exons 4 et 5 a été démontrée [13]. Chez la souris, le gène se situe sur le chromosome 7 et sur le chromosome 11 (11q14-21) chez l'homme

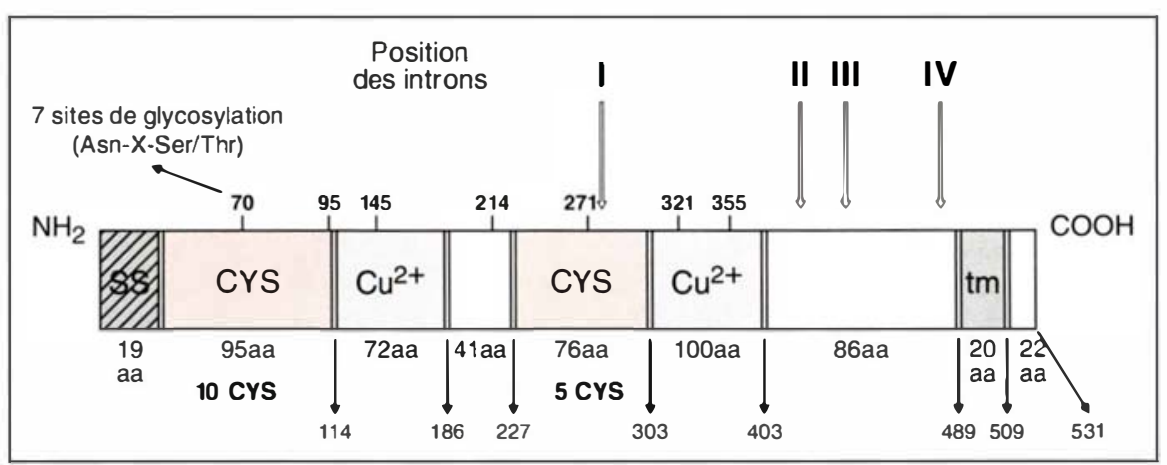

Figure 2. Structure de la tyrosinase. La molécule de tyrosinase code pour un polypeptide de 531 acides aminés, dont le poids moléculaire serait de $60 \mathrm{kDa}$. La protéine glycosylée a un poids moléculaire d'environ $70 \mathrm{kDa}$. tm : domaine transmembranaire. SS : séquence signal.

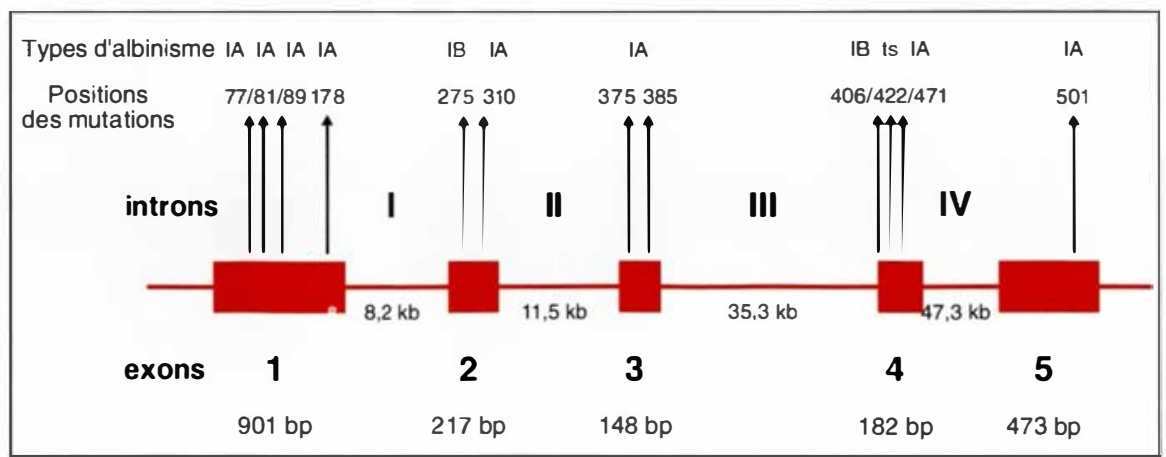

Figure 3. Gène de la tyrosinase. Le gène de la tyrosinase est situé sur le chromosome 11 (11q14-21) chez l'homme et sur le chromosome 7 chez la souris, dans des régions synténiques. 


\section{RÉFÉRENCES}

15. Murty VVVS, Bouchard B, Mathew S Vijayasaradhi S, Houghton AN. Assignment of the human TYRP (brown) locus to chromosome region 9 p23 by non radioactive in situ hybridization. Genomics $1992 ; 13$ 227-9.

16. Kluppcl M, Beermann F, Ruppert S, Schmid E, Hummler E, Schutz G. The mouse tyrosinase promoter is sufficient for cxpression in melanocytes and in the pig mented epithclium of the retina. Proc Nat Acad Sci USA 1991; 88 : 3777-81.

17. King RA, Olds IPP. Hairbulb tyrosinasc activity in oculocutancous albinism suggestions for pathway control and block location. Am J Med Genet 1985 ; 20 : 49-55

18. Gicbel LB, Strunk KM, King RA, Hanifin JM, Spritz RA. A frequent tyrosinasc gene mutation in classic, tyrosinasencgative (typc IA) oculocutancous albinism. Proc Natl Acad Sci USA 1990 ; 87 : 3255-8.

19. Tomita $\mathrm{Y}$, Takeda A, Okinaga S, Tagami H, Shibahara S. Human oculo cutancous albinism caused by a single basc insertion in the tyrosinasc gene. Biochem Biophys Res Commun 1989; 164: 990-6.

20. Gicbcl I,B, Tripathi RK, King RA, Spritz RA. A tyrosinase gene missense mutation in temperature-sensitive type I oculocutancous albinism. A human homologuc to the siamesc cat the himalayan mousc. J Clin Invest 1991; 87 : 1119-22.

21. Giebcl LB, Tripathi RK, Strunk KM, Hanifin JM, Jackson CE, King RA, Spritz RA. Tyrosinase gene mutations associated with typc IB (yellow) oculocutancous albinism. Am J Hum Genet 1991; 48 : 1159-67.

22. Zdarsky E, Favor J, Jackson IJ. The molecular basis of brown, an old mousc mutation and of a revertant to wild type. Genetics 1990 ; 126 : 443-9.

23. Jackson IJ. A cDNA cncoding tyrosinasc-related protein maps to the brown locus. Proc Natl Acad Sci USA 1988; 85 : 4392-6.

24. Hearing VJ, Jimencz M. Analysis of mammalian pigmentation at the molccular lcvel. Pig Cell Res 1989 ; 2 : 75-85

25. Halaban R, Moellmann G. Murina and human $b$ locus pigmentation genes encode a glycoprotcin (gp75) with catalase activity Proc Natl Acad Sci USA 1990 ; 87 : 4809-13.

26. Vijayasaradhi S, Houghton AN. Purification of an autoantigenic $75 \mathrm{kDa}$ human mclanosomal glycoprotein. Int $J$ Cancer $1991 ; 4$ : 298-303.

27. Johnsdon R, Jackson IJJ. Light is a dominant mousc mutation resulting in premature ccll death. Nature Genet 1992; 1 : 226-9.
$[14,15]$. Il faut noter que ces chromosomes sont homologues entre les deux espèces.

La structure de la région promotricc du gène de la tyrosinase a ćgalement ćté caractérisće. Un minigène construit avec $2,5 \mathrm{~kb}$ de la séquence située en amont du gène, incluant le promoteur, liée à l'ADNc spécifique induit une pigmentation chez des souris transgéniques. Cette pigmentation se caractérise par une expression " tissu-spécifique " des mélanocytes " [16]. On peut à présent envisager d'analyser les éléments régulateurs (promoteurs et enhancers) nécessaires à cette expression spécifique ainsi que les interactions molćculaires contrôlant l'expression de ce gène.

\section{Génétique de l'albinisme}

Lc terme albinisme regroupe un ensemble de maladies génétiques, transmises de façon autosomique et récessive, qui se caractérisent par l'absence totale ou partielle de synthèse des mélanines [17] (Tableau I). La forme la plus sévère de cette maladie est l'albinisme à tyrosinase négative ou albinisme de type IA, dans lequel aucunc activité n'est détectée dans les yeux, la peau ou les cheveux. L'absence de pigmentation peut conduire à des troubles oculaires, dus à des défauts du nerf optique et prédispose les sujets atteints à certains cancers de la peau. L'isolement du gène de la tyrosinase a soulevé un grand intérêt chez les scientifiques afin de définir les mutations qui affectent l'activité tyrosinase chez l'homme. A ce jour, de nombreuses mutations ont ćté décrites. Ce sont toujours des mutations ponctuelles, dont certaines sont faux-sens alors que d'autres provoquent des modifications du cadre de lecture. Ces mutations peuvent se produire dans différentes régions de la molćcule et conduisent toutes à la disparition de l'activité enzymatique, et donc de la production de mélanine.

Ainsi, on peut mentionner la mutation décrite au codon 81 (1 $1^{\text {er }}$ exon) changeant l'acide aminé proline en leucine (CCT devient CTT) [18]. Bicn que cette mutation n'affecte aucune des régions critiques de la moléculc (peptide signal, région transmembranaire ou sites de glycosylation), elle inactive la tyrosinase ainsi produite. Un autre exemple est l'introduction d'un codon stop en position 316 à la suite d'un décalage de phase de lecture secondaire à l'insertion d'un résidu $\mathrm{C}$ en position 310 [19] (CCA devient CCCA). Signalons aussi le cas de la mutation " température-sensible ", correspondant à la mutation siamoise chez le chat et à la mutation himalayan chez la souris. En effet, les sujets atteints de cette forme d'albinisme particulier présentent des poils pigmentés dans les zones corporelles dont la température moyenne est plus faible (bras et jambes), alors que le reste du corps présente des poils blancs. La tyrosinase de ces patients a la particularité d'être inactivée aux températures supérieures à $35^{\circ} \mathrm{C}$. Cette mutation a été localisée au codon 422 (exon IV) et résulte de la permutation CGG en CAG (l'acide aminé arginine devient une glutamine) [20].

\section{Tableau}

ALBINISME

Groupe de maladies génétiques affectant la formation de mélanine

- Albinisme oculaire

- Albinisme oculo-cutané (OCA) Type IA OCA : tyrosinase négative Absence de mélanine dans la peau, les yeux, les cheveux Type IB OCA : " jaune " Absence de mélanine à la naissance. Apparition de phaeomélanine Type III : complexes génétiques avec différents allèles anormaux. Pigmentation de l'iris

Type sensible à la température 
Plusicurs mutations ont également été caractérisées dans les cas d'albinisme de type IB. Dans cette deuxième forme de la maladie, le pigment n'est pas détectable à la naissance, mais il y a accumulation d'une faible quantité de mélanine de type phacomélanine au cours de l'enfance et à l'âge adulte. Principalement deux mutations ont été identifiées pour les formes IB. Il s'agit des substitutions aux codons 275 et 406 [21] (Tableau II). Chez les patients atteints d'albinisme de type III (ou albinisme à pigment minimal), il y a absence de pigment dans la peau et les cheveux, mais ces individus développent cependant une pigmentation de l'iris au cours des dix premières années de leur vie. Il est probable que les différentes formes d'albinisme oculocutané résultent de la présence d'une ou plusieurs des différentes mutations déjà décrites.

Ainsi, chez l'homme, l'isolement du gène de la tyrosinase permet d'envisager le diagnostic prénatal et la détection des individus porteurs; cependant, l'hétérogénéité des mutations décrites au locus albino rend nécessaire l'utilisation de sondes multiples correspondant à toutes les régions de la molécule de tyrosinase.

\section{Autres gènes et protéines spécifiques de la mélanogenèse}

\section{TRP-1 (locus brown)}

Parmi les clones d'ADNc spécifiques des mélanocytes, l'un des candidats initialement proposé pour le gène de la tyrosinase a en fait été localisé sur le chromosome 4 chez la souris, au locus brown. Comme le locus albino, le locus brown a été bien étudié par les généticiens [22]. En effet, les mutations observées à ce locus entraînent la production de mélanine brune et non pas noire. C'est pourquoi on attribue à la protéine brown un rôle important dans la détermination du type de mélanine produit, noir ou marron. Cette protéine est similaire à la tyrosinase bien qu'elle en soit clairement distincte. Elle présente un degré d'homologie de $45 \%$ de séquence avec la molécule de tyrosinase, et sa structure est conservée entre la souris et l'homme. En raison de cette similarité, la protéine du locus brown a été dénomméc TRP-1 (tyrosinase-related-protein-1) [23]. Plusicurs hypothèses ont été émises quant au rôle précis du locus brown dans la mélanogenèse. Parmi cellesci, certains auteurs n'écartent pas une activité tyrosinase complémentaire qui agirait en synergie avec le gène du locus albino [24]. D'autres auteurs attribuent à TRP-1 une activité catalase [25], qui pourrait protéger la mélanine des effets du peroxide d'hydrogène, l'une des substances toxiques produites au cours de la mélanogenèse. Chez l'homme, la protéine, également appeléc gp75, a été identifiée comme un auto-antigène présent chez certains sujets atteints de mélanomes malins [26]. C'est également la protéine la plus abondante dans les cellules mélanocytaires. Son expression est parfaitement corrélée à la présence de la mélanine. Cela n'est

Tableau II

MUTATIONS ASSOCIÉES AVEC L'ALBINISME DE TYPE I

\begin{tabular}{|rrlllll|}
\hline Codon & Exon & \multicolumn{5}{c|}{ Substitution } \\
\hline 77 & I & CGG & CAG & Arg & GIn & Type \\
81 & I & CCT & CTT & Pro & Leu & IA \\
89 & I & TGC & CGC & Cys & Arg & IA \\
178 & I & TGG & TAG & Trp & STOP & IA \\
275 & II & GTC & TTC & Val & Phe & IB \\
310 & II & CCA & CCCA & STOP & 316 & IA \\
375 & III & ACA & AAA & Thr & Lys & IA \\
385 & III & GAT & AAT & Asp & Asn & IA \\
406 & IV & CCT & CTT & Pro & Leu & IB \\
422 & IV & CGG & CAG & Arg & GIn & ts \\
471 & IV & GTT & GTTC & STOP & 490 & IA \\
501 & V & CTG & CTTG & Val & STOP & IA \\
\hline
\end{tabular}

$m / s n^{\circ} 4$ vol. 9, auril 93 pas toujours le cas pour la tyrosinase codée par le locus albino, puisque des cellules non pigmentées peuvent exprimer le transcrit spécifique de la tyrosinase en quantité non négligeable. Le gène du locus brown a été isolé chez l'homme et chez la souris. Sa structure est conservée au cours de l'évolution, mais elle est assez différente de celle du gène de la tyrosinase, à la fois en ce qui concerne sa taille (15 kb au lieu de 70) et son organisation (huit introns au licu de quatre pour le gène de la tyrosinase) (Bouchard et al., soumis pour publication). Le gène de TRP-1 a été localisé sur le chromosome 9 dans la région $9 \mathrm{p} 23$ [15]. Cette localisation a permis de définir une région synténique avec le chromosome 4 chez la souris. Comme nous l'avons vu, plusieurs mutations existent chez la souris, la plus remarquable d'entre elles a été caractérisée : elle donne lieu à la formation de mélanine brune et est causée par le remplacement d'un résidu cystéine par une tyrosine. Chez l'homme, aucune mutation n'a été décrite à ce locus, mais les travaux récents de Jackson et al. [27] semblent suggérer le rôle possible d'une telle mutation dans le grisonnement précoce (ou canitic) observé chez certains individus. La toxicité de la mélanine ainsi produite pourrait être à l'origine de la mort précoce des mélanocytes affectés par cette mutation.

\section{TRP-2 (locus slaty)}

Un troisième clone d'ADNc isolé de façon concomitante avec la tyrosinase et TRP-1, et cela par des méthodes analogues (criblage d'une banque d'ADNc avec des anticorps polyclonaux) a été caractérisé récemment [28]. Là encore, cette molécule présente une homologie de structure avec la tyrosinase et TRP-1. Chez la souris, ce clone a été localisé sur le locus slaty, sur le chromosome 14 , et son rôle dans la mélanogenèse semble avoir été élucidé. Il s'agit en effet de la DOPAchrome tautomérase, autre enzyme essentielle de la mélanogenèse. En effet, cette enzyme isomérise le DOPAchrome en DHICA (5,6-dihydroxyindole-2-carboxylic acid) (figure 1). En l'absence de DOPAchrome tautomérase, le DOPAchrome forme spontanément un produit 
distinct, le DHI (5,6-dihydroxyindole). Les mélanines dérivées de DHICA ou de DHI ont des propriétés différentes (solubilité, aspect et couleur). La DOPAchrome tautomérase représente donc un élément de contrôle important qui règle le type de mélanine produite.

\section{Pmel 17-1 (locus silver)}

Enfin, un quatrième clone présentant une homologie de structure avec les TRP a été initialement appelé Pmel 17-1 [29]. L'expression de ce gène est également restreinte aux mélanocytes. La protéine a un poids moléculaire de $70 \mathrm{kDa}$, possède une région transmembranaire et plusieurs sites potentiels de glycosylation.

Pmel 17-1 est conservée entre l'homme et la souris, et a pu être associée, par des études de recombinaison génétique, avec le locus silver. Les souris atteintes de cette mutation présentent une hypopigmentation, et une perte prématurée des mélanocytes présents dans le follicule pileux. L'expression de Pmel 17-1 est corrélée à la quantité de mélanine synthétisée. Jusqu'à présent, sa fonction est inconnue, mais ces observations indiquent qu'elle pourrait également avoir une activité mélanogénique.

\section{Conclusion}

Les similarités existant entre les tyrosinases murines et humaines et les TRP définissent les régions importantes dans la fonction de ces molécules. Ces régions consistent entre autres, comme nous l'avons vu, en domaines riches en résidus cystéines et en une région transmembranaire hydrophobe à l'extrémité C-terminale, correspondant à l'intégration des protéines dans la membrane des mélanosomes. De plus, ces molécules contiennent toutes deux des régions présentant une homologie avec le site de liaison $\mathrm{au} \mathrm{Cu}^{2+}$ des hémaocyanines. Il est tout à fait remarquable qu'avec l'apport de techniques biochimiques et moléculaires, quatre gènes différents aient pu être isolés au cours de ces dernières années, possédant une telle homologie de structure et qui semblent être conservés au cours de l'évolution.

L'une des voies de recherche actuelle dans l'étude de la mélanogenèse porte sur l'identification et la caractérisation d'autres molécules éventuelles appartenant à cette même famille. Par ailleurs, de nombreuses questions restent à résoudre, parmi lesquelles : quelles sont les interactions entre ces différentes molécules au cours de la mélanogenèse, et comment chacune d'entre elles participe-t-elle aux différentes étapes de la mélanisation? Nous commençons à entrevoir certains des mécanismes contrôlant l'expression de ces gènes. La connaissance des séquences promotrices devrait ouvrir la voie à des travaux permettant de déterminer les " motifs" responsables de cette régulation et de mieux comprendre le rôle des différents inhibiteurs et stimulateurs de la mélanogenèse. Une autre question concerne les signaux qui "guident " les protéines mélanosomales impliquées dans la mélanogenèse vers le lieu de leur action enzymatique. Existe-t-il des signaux ou séquences spécifiques qui contrôlent le passage de ces molécules à travers l'appareil de Golgi ? Quelle est la séquence des événements permettant la mise en place des activités cellulaires de ces différentes molécules, et la synthèse des mélanines ?

On sait par ailleurs que de très nombreux intermédiaires de la mélanogenèse peuvent se révéler toxiques pour la cellule s'ils sont déversés dans le cytoplasme. Les mécanismes permettant à la cellule de se protéger contre ces médiateurs jouent sans doute un rôle très important dans la mélanogenèse. De nombreux aspects de santé publique sont liés à une meilleure connaissance du rôle des rayons UV et de la lumière solaire. Plusieurs études ont montré l'augmentation dramatique de l'incidence des cancers de la peau liés directement à l'exposition aux rayons solaires. La compréhension des mécanismes cellulaires et moléculaires contrôlant la production de mélanine par l'organisme est donc cruciale dans le contexte de la lutte contre de nombreuses maladies environnementales. Enfin, comme nous l'avons vu, on peut s'attendre à la compréhension de nouvelles affections liées à ces gènes récemment caractérisés. Ces études sont cruciales pour le dépistage de maladies génétiques liées ou associées aux troubles de la pigmentation

\section{RÉFÉRENCES}

28. Jackson IJJ, Chambers DM, Tsukamoto K, Copeland NG, Gilbert DJ, Jenkins NA, Hearing V. A second tyrosinase related protein, TRP-2, maps at the slaty locus. EMBO J $1992 ; 11: 527-35$

29. Kwon B, Chintamaneni C, Kozak C, Copeland NG, Gilbert DJ, Jenkins N, Barton D, Francke U, Kobayashi Y, Kim KK. A melanocyte spccific gene, Pmel 17 maps near the silver coat color locus on mousc chromosome 10 and is in a syntenic region on human chromosome 12. Proc Natl Acad Sci USA 1991; 88 : 9228-35.

\section{Summary}

Proteins regulating mclanogenesis. Molecular genetics of cutaneous albinism

Pigmentation - or melanogenesis - results from interactions between several genes and their products, to produce melanins. These specific pigments are synthesized by melanocytes, within specialized organelles, the melanosomes. Melanogenesis is regulated at many different levels, developmental, cellular as well as molecular. However, one enzyme, tyrosinase, is mainly responsible for the control of melanin biosynthesis. Previous work using mice genetics had shown linkage between the tyrosinase gene and the mouse albino locus. The structure of this protein has now been elucidated, its gene cloned, and several mutations have been characterized in human and mice. These mutations are responsible for the different forms of albinism. More recently, other melanocyte-specific genes have been cloned and characterized. The structure of these genes and their products is related to that of tyrosinase, and therefore defines a family of molecules which interactions are essential for the cellular events of melanogenesis.

\section{TIRÉS A PART}

B. Bouchard 